\title{
The optimization of a distribution and over distribution line structure
}

\author{
S. Firouzifar E.Dahlquist \\ Malardalen University, Vasteras, Sweden, s firouzifar@ yahoo.com
}

\begin{abstract}
This paper discusses that the significant voltage drop under load condition is the big problem of distribution networks, because it limits the transfer capability of distribution lines. A utilization of the compact lines technology allows a significant increase in the transfer capability of distribution lines. Important developments for controlling over voltage and conductor resonances in recent decades gave the possibility to reduce the distances between phases. The modern distribution lines can be optimized with respect to their electric parameters in comparison with normal distribution lines, and by this increase the capacity in energy transmission.
\end{abstract}

Keywords: Natural power, transmitted power, capacity

\section{Nomenclature}

\begin{tabular}{|c|c|}
\hline$P_{n}=S I L$ & surge impedance loading \\
\hline$U_{1}$ & voltage at the start of line \\
\hline$U_{2}$ & voltage at the end of line \\
\hline$\lambda$ & line impedance $\lambda=w l / v$ \\
\hline J & current density, $\mathrm{A} / \mathrm{mm}^{2}$ \\
\hline$\rho$ & conductor special resistance \\
\hline$\ell_{c r}$ & length of line \\
\hline$x$ & inductive impedance \\
\hline$R$ & active resistance \\
\hline$P / P n$ & $\begin{array}{l}\text { transmitted power / surge impedance } \\
\text { loading or natural power }\end{array}$ \\
\hline$U_{2 . n o m}$ & real value voltage \\
\hline$\varphi$ & shift angle between voltage and current \\
\hline Dav & $\begin{array}{l}\text { average geometrical distance between } \\
\text { conductors }\end{array}$ \\
\hline$Z$ & surge impedance \\
\hline$r_{0}$ & radius of a conductor \\
\hline Irc & $\begin{array}{l}\text { reactive current end of line for } \\
\text { consumed }\end{array}$ \\
\hline$I_{2}$ & loading current end of a line \\
\hline
\end{tabular}

\section{Introduction}

Voltage drops occur in transmission lines, sub transmission lines and distribution lines between the source and load. The voltage drop is very important when the impedance of transmission and subtransmission line is high relative to the components of the circuit. By choosing suitable physical inductive reactive power and capacity power in exit line. Then we do not need to have extra equipment like capacitors and reactors. The high efficient transmission of electricity is depending on two important problems: voltage drop and power losses. Since alternating current $(A C)$ depend on: (1) reactive power, and (2) characteristic impedance (inductance and capacitance).

Part of the capacity transmitted depends on reduced reactive power in the line. If we provide the right conditions, electrical energy can be transmitted without power losses. The reactive power can be compensated by extra equipment installed in the line as a parallel reactor or compensating capacitor, but we will have problems to provide equipment and we have to pay a lot of money for the purchase of the equipment. There are other methods for reducing reactive power by creating balance between reactive power, inductance and capacitance in the line. This will be discussed in this paper.

The effect cost for these equipment can be predicted, respectively. Transmission line design is discussed in (Clerci and Landonin, 1991; Heidari and Heidari, 2002; Doss, 2002). More details of technology is available e.g. in (Alcola Conduc tor Accessories, 2003; ACCR, 2003; 3M, 2003).

\section{Designing modern lines in sub- transmission and distribution voltages}

The equivalent scheme of a distribution lines is presented in Figure 1, Here $X$ is the inductive impedance of a line, $R$ is its active resistance. Let us present the loading current at the consuming end of a line in the symbolized form

$$
I_{2}=I_{n} \frac{P}{P_{n}}(1-j \cdot \operatorname{tg} \varphi)
$$




$$
I_{n}=\frac{U_{2 . n o m}}{Z}
$$

where $Z$ is the surge impedance of a line, $P_{n}$ is the natural power of this line or surge impedance loading, $\mathrm{P}$ is the transmitted power, $\varphi$ is the shift angle between voltage and current. The reactive current, consumed (generated) by the line at its end is equal to

$$
I_{r . e}=j \frac{U_{2, n o m k}}{2 Z} \frac{X}{Z}\left[1-\left(\frac{P}{P_{n}}\right)^{2}\right]=j I_{n} \frac{\lambda}{2}\left[1-\left(\frac{P}{P_{n}}\right)^{2}\right] \text {, }
$$

where $\lambda$ is the wave length of a line. By $P=P_{n}$, the line does not consume and does not generate reactive current. But when $P<P_{n}$ the line generates a reactive current, and when $P>P_{n}$, the line consumes reactive current. The sum of a current at the end of a consuming end of a line is equal to

$$
\dot{I}=\frac{U_{2, \text { nom }}}{Z} \frac{P}{P_{n}}(1-j \cdot \operatorname{tg} \varphi)+j \frac{U_{2 \text { nom }}}{Z} \frac{\lambda}{2}\left[1-\left(\frac{P}{P_{n}}\right)^{2}\right] \text {, }
$$

The voltage at the sending end of a line is shown in Figure 2.

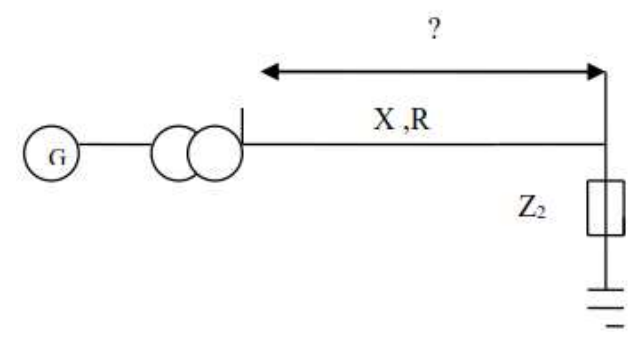

Figure 1. Inductive impedance $(\mathrm{X})$ and active resistance (R) in distribution line.

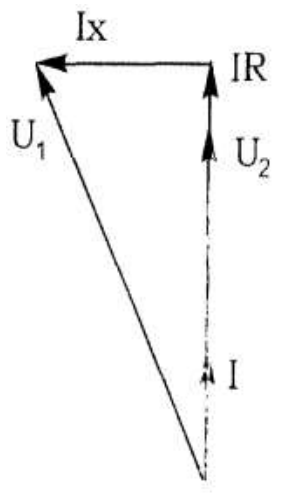

(a)

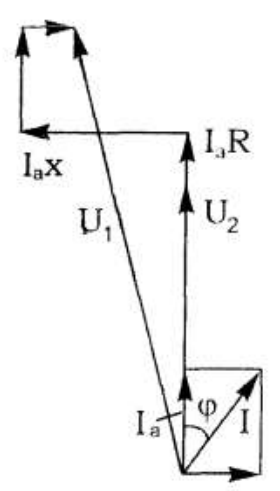

(b)
Figure 2. Vector diagram of voltages for transmission lines (a) for clean active loading and by presence of inductive component (b).

$$
\begin{aligned}
& \dot{U}=U_{2}+I(R \dot{+} j X)= \\
& =U_{2}+U_{2 \max }\left\{\frac{P}{P_{n}}\left(\frac{R}{Z}+\frac{X}{Z} \operatorname{tg} \varphi\right)+\left[\left(\frac{P}{P_{n}}\right)^{2}-1\right] \frac{X}{Z} \frac{\lambda}{2}+j \frac{P}{P_{n}}\left(\frac{X}{Z}-\frac{R}{Z} \operatorname{tg} \varphi\right)\right\}= \\
& =U_{2}+U_{2 \operatorname{man}}\left\{\frac{P}{P_{n}}\left(\frac{R}{Z}+\lambda \operatorname{tg} \varphi\right)+\left[\left(\frac{P}{P_{n}}\right)^{2}-1\right] \frac{\lambda^{2}}{2}+j \frac{P}{P_{n}}\left(\lambda-\frac{R}{Z} \operatorname{tg} \varphi\right)\right\}=
\end{aligned}
$$

where vector $U_{2}$ is combined with the axis of a real value and $X=\lambda Z$. By neglecting the unreal component, which influences on the value of $U_{1}$ practically negligible and by assuming that $U_{2}=U_{2 . n o m}$ we obtain the value of voltage at the sending end of a line:

$$
U_{1}=U_{2}\left\{1+\frac{P}{P_{n}}\left(\frac{R}{Z}+\lambda \cdot \operatorname{tg} \varphi\right)+\left[\left(\frac{P}{P_{n}}\right)^{2}-1\right] \frac{\lambda^{2}}{2}\right\},
$$

where the assumption $\left(U_{2}=U_{2 . n o m}\right)$ is real, because by permissible voltage drop the voltage at the consuming end is to be not less then $U_{2 . n o m}$.

The ratio $R / Z$ can be estimated by the next method. The active cross-section of the phase conductor is equal to

$$
F_{a}=\frac{I}{J}=\frac{I_{a}}{J \cdot \cos \varphi}=\frac{I_{a}}{J} \frac{P}{P_{n}} \frac{1}{\cos \varphi}=\frac{U_{2 \text { nom }}}{J Z} \frac{P}{P_{n}} \frac{1}{\cos \varphi},
$$

where $J$ is the current density in a conductor. Hence the active resistance of the phase conductor is equal to

$$
R=\frac{\rho \cdot \ell}{F_{a}}=\frac{\rho \ell \cdot z \cdot J}{U_{2}} \frac{P_{n}}{P} \cos \varphi,
$$

where $\rho$ is the specific resistance of a conductors and / is the length of a line. Therefore, the ratio

$$
\frac{R}{Z}=\frac{\rho \cdot \ell \cdot z \cdot J}{U_{0}} \frac{P_{n}}{P} \cos \varphi
$$

Putting this ratio into the relation (6) we obtain

$U_{1}=U_{2}+\rho \cdot J \cdot \ell \cos \varphi+U_{2} \frac{P}{P_{n}} \lambda_{n} \operatorname{tg} \varphi+U_{2}\left[\left(\frac{P}{P_{n}}\right)^{2}-1\right\rceil \frac{\lambda^{2}}{2}$,

or the square equation in relation to the ratio $P / P_{n}$

$$
\left(\frac{P}{P_{n}}\right)^{2} \lambda^{z}+2 \frac{P}{P_{*}} \lambda \cdot \operatorname{tg} \varphi+2+\frac{2 \rho \cdot J \cdot \ell \cdot \cos \varphi}{U_{2}}-2 \frac{U_{1}}{U_{1}}-\lambda^{z}=0,
$$

As a result of this equation solution the permissible ratio $P / P_{n}$ by the given permissible ratio $U_{1} / U_{2}$ is equal to

$$
\frac{P}{P_{n}}=\frac{1}{\lambda}\left[\sqrt{\operatorname{tg} \varphi^{2}+2\left(\frac{U_{1}}{U_{2}}-1-\frac{\rho J \cdot \cdot \cos \varphi}{U_{2}}+\lambda^{2}\right)}-\operatorname{tg} \varphi\right],
$$


Inserting in the last formula we get a value of $S I L$

$$
P_{n}=\frac{3 U_{2, \text { maen }}^{-}}{Z},
$$

We obtain another formula for the permissible transmitted power over a line

$$
P=\frac{3 U_{2}^{2}}{Z}\left[\sqrt{\operatorname{tg} \varphi^{2}+2\left(\frac{U_{1}}{U_{2}}-1-\frac{\rho \cdot J \cdot \ell \cdot \cos \varphi}{U_{2}}+\lambda^{2}\right)}-\operatorname{tg} \varphi\right]
$$

It is possible to conclude from these last formulas, that it is impossible to transmit electrical energy over relatively short lines $(\lambda \leq 0.1 \mathrm{rad})$, for which these formulas were obtained, without voltage drop. More over the voltage drop along the line is to be bigger than the voltage drops on the active resistance, which is determined by the item with the specific resistance $\rho$.

By the presence of an inductive load $(\varphi>0)$, the total voltage drop over the line is to be cover and increasing the reactive item, which is determined by the last item in (12) and (14). Therefore by nominal operating voltage at the consuming end of a line the voltage at the sending end of a line is to be bigger, however, not bigger than the maximum operating voltage. For this reason the bigger is the length of a line, the less is the permissible transmitted power. By the given permissible ratio $U_{1} / U_{2}$ and by the given length of a line, the permissible transmitted power $P$ is inverse proportional to the surge impedance of a line.

\section{Identification of surge impedance in modern distribution lines}

This conclusion confines the efficiency of measures for compactization of a line structure and additional measures, caused the decrease of the surge impedance of a line. Really the surge impedance of lines with single conductors in a phase is equal to

$$
Z=60 \ell n \frac{D a v \cdot g}{r_{0}},
$$

where Dav.g is the average geometrical distance between all three phases, $r_{0}$ is the radius of a conductor. By the decrease of Dav.g. The surge impedance $Z$ decreases, but not so much. It is possible to decrease $Z$ by using a conductor bundle instead of a single conductor with the same cross-section which is determined by the selected value of $J$. For double conductors in a phase the surge impedance of a line is equal to

$$
Z=60 \ln \frac{D a v \cdot g}{\sqrt{r_{0} d}}
$$

where $d$ is the distance between two sub conductors. By triple conductors in a phase, the surge impedance of a line is equal to

$$
Z=60 \ln \frac{\text { Dav.g }}{\sqrt[3]{r_{0} d^{2}}}
$$

and by four sub conductors

$$
Z=60 \ell n \frac{\text { Dav.g }}{\sqrt[4]{r_{0} d^{3} \cdot \sqrt{2}}}
$$

It is possible to see that the bigger the number of sub conductors the more effective is the splitting of the conductor. It is necessary to note that the efficiency of a conductor splitting increases by the decrease of Dav.g. The minimum Dav.g is by the triangle disposition of all phases.

By optimizing the distance between neighboring conductors $d=0,5-0,6 \mathrm{~cm}$ and by minimum possible distances between phases it is possible to decrease the surge impedance of a distribution line by two - three times compared to conventional lines (130 - $160 \mathrm{Ohm}$ instead of $350-400 \mathrm{Ohm})$. Without interphases, the insulation spacers (compact lines) are two times and approximately three times less by the installation of interphase insulation spacers (super compact lines). This is in accordance with (14).

\section{Ratio $P / P_{n}$ versus length}

The permissible ratio $P / P_{n}$, which was calculated by means of formula (12) with permissible voltage drop $U_{l} / U_{2}=1.1$ by current density $\mathrm{J}=1 \mathrm{~A} / \mathrm{mm}^{2}$, by different values of a power factor $\cos \varphi$ and by different operating voltages of lines are presented in Figure 4 versus their length.

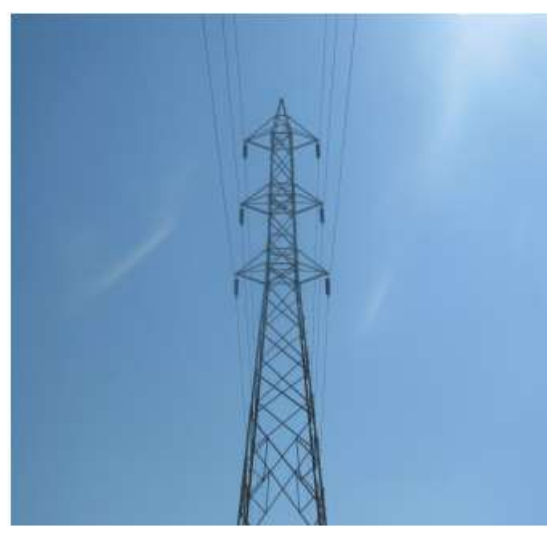

Figure 3. Voltage line.

As it is possible to see for each level of operating voltage a decrease of the power factor $\cos \varphi$ leads to a significant decrease of permissible ratio $P / P_{n}$, if this ratio $P / P_{n}>1$. On the contrary if this ratio $P / P_{n}<1$ the decrease of $\cos \varphi$ leads to the increase of a permissible ratio $P / P_{n}$. A crossing of all curves takes 
place by the same length of lines. This critical length of lines $\ell_{\mathrm{cr}}$ increases when the operating voltage increases: The dependence $\ell_{c r}=f\left(U_{\text {nom }}\right)$ is linear (Table 1) and can be estimated by means of the simple formula

$$
\ell_{c r}=1.9 U_{\text {nom }},
$$

where $\ell_{\mathrm{cr}}$ is in $\mathrm{km}$ and $U_{\text {nom }}$ is in $\mathrm{kV}$.
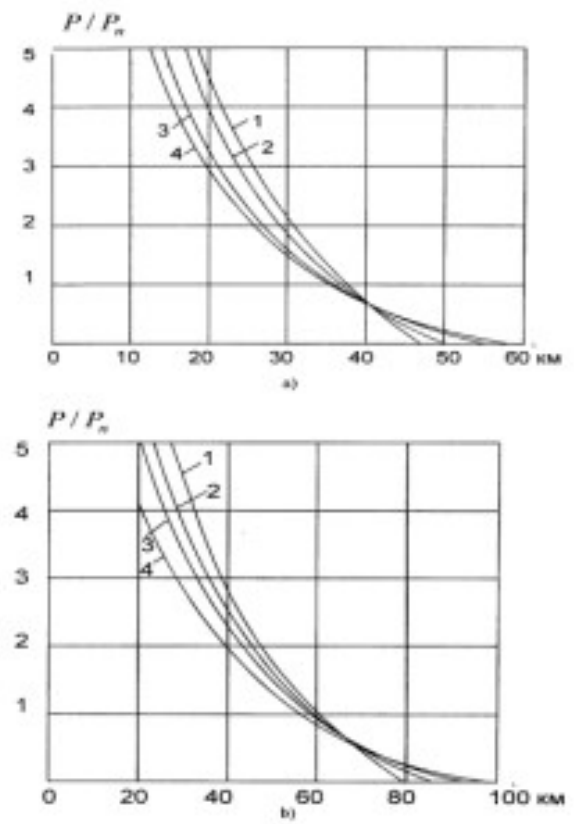

Figure 4. Ratio $P / P_{n}$ versus length in kilometers.

An increase of the operating voltage leads to a significant increase of the permissible ratio $P / P n$ for the given length of a line. Respectively, the permissible length of a line for a transmission of the given ratio $P$ / $P n$ increases significantly by an increase in the operating voltage. Table 1 shows the significant length of modern distribution line for different operating voltages

Table 1. Operating voltages.

\begin{tabular}{lrrr}
\hline$U_{\text {nom }}, \mathrm{kV}$ & 20 & 35 & 63 \\
\hline$\ell_{c r}, \mathrm{~km}$ & 39,5 & 65 & 120 \\
\hline
\end{tabular}

\section{Conclusions}

Low power factor leads to the increase of voltage drop along the line and as the result limits the transmitted power and the possible length of electrical energy transmission. In order to decrease the influence of the power factor on the transfer capability of lines it is very useful to decrease inductive impedance of lines by using bundles of conductors and decreased distances between phases.

\section{References}

ACCR. Power Conductor Accelerated Testing Facility (PCAT), ACCR Technology Description, November 2003.

Alcola Conductor Accessories. Transmission Accessories, 2003.

G. N. Alexandrov and H. Heidari. Equalization of overhead Ch. RI, "Heida" Increasing Lind Voltage.

Aluminum Conductor Composite Reinforced, Technical Notebook, 3M, March 2003.

A. Chakir and H. Koch. Thermal Calculation for Buried Gas -Insulated Transmission Lines (GIL) and XLPE-Cale, In IEEE Winter Power Meeting 2001, Columbus, 2001.

A. Chakir and H. Koch. Long Term Test of Buried Gas Insulated Transmission Lines (GIL). In IEEEWPM 2002 New York, 2002.

A. Clerci and M. Landonio. EHV Compact Lines - a new solution. In CIGRE, 1991.

CRIEPI, Report: Development of Make 66 to $154 \mathrm{kV}$ Overhead Compact Transmission Lines (Part7) - Study on Mechanical Stresses to $154 \mathrm{kV}$ Insulation Arms on FullScale Actual Test. CRIEPI Rep. W95037

Dennis Doss. Double your transmission capacity without changing existing towers. In Electric light 7 and Cable, August 2002.

Gh. Heidari. Equalization of overhead transmission line capacity different classes of voltage. In PSC 95, Tehran, Nov. 1995

Gh. Heidari and G. N. Alexandrov, Increasing line voltage or sub conductors number each phases. In CIGRE, Paris, France, Sept. 1996.

Gh. Heidari and Maziar Heidari. Effect of land price on transmission line design. In CIGRE, Sept. 2002.

C. G. Heningen, G. Kaul, H. Koch, A. Scheuette, and R.Plath. Electrical and Mechanical Long-Time Behaviour of Gas Insulated Transmission Lines. In CIGRE, 08/2000.

T. Hiller and H. Koch. Gas Insulated Transmission Lines (GIL): proven technology with new performance. In Xiamen Switchgear Seminar, China, 1997

T. Hiller and H. Koch. Gas-Insulated Transmission Lines for high power transmission over long distances. In EMPT, Singapore, 1998a.

T. Hiller and H. Koch. Gas -insulated Transmission Lines (GIL): A solution for the power supply of metropolitan areas. In CEPSI, Thailand, $1998 \mathrm{~b}$.

H. Koch and A. Schuette. Gas Insulated Transmission over long distances. In EPSR, Hong Kong, 1997.

Inventions \& Innovations. Development of a CompositeReinforced Aluminum Conductor, Project Fact Sheet, 1999.

H. B. Markus, P. Vestner, Diego F. Sologuren-Sanchez, Bodo A. Bruhl, and Paul F. Coventry. Dimensioning criteria and test result for a polymer enclosed gas insulted line. In CIGRE, 2000.

F. Sganzerla, J. A. A., Casagrande, and D. B. Galia. Electron are Brazila $-500 \mathrm{kV}$ and $230 \mathrm{kV}$ Compavr Lines: Design and 2. G or subconductors number in each phase, CIGRE, Paris, France, Sept.1996. 


\section{Biography}

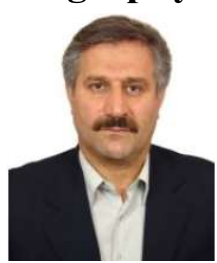

Sohrab Firouzifar was born in Damavand in Tehran, IRAN, on July 9, 1959. He graduated from The Institute of Technology of Tehran (M.Sc.) and is a $\mathrm{PhD}$ student at Malardalen University in Vasteras Sweden. He was the director of standard and quality control in the Mazandaran regional electrical company in the north of Iran and is a member IEEE in IRAN, and member of the board in IEEE north of Iran. He has published several papers in Iran for example: International Power system conference (PSC) and distribution system conference about expert systems in transmission substation \& power transformer utilization. $\mathrm{He}$ was the director for the technical office for 10 years and was director R\&D in M.R.E.C for 7 years. 